\title{
Análise das complicações em pacientes no período de recuperação anestésica*
}

\author{
Analysis of patient complications in the post-anesthesia recovery \\ Análisis de las complicaciones en pacientes durante el período de recuperación anestésica
}

Fiama Chagas Nunes ${ }^{1}$, Selme Silqueira de Matos², Ana Lúcia De Mattia ${ }^{3}$

RESUMO: Objetivo: Analisar as complicações do paciente em período de recuperação anestésica. Método: Trata-se de uma pesquisa com abordagem metodológica quantitativa, delineamento não experimental, comparativa, de campo e prospectiva. A amostra foi constituída por 42 adultos, submetidos à cirurgia eletiva, com anestesia geral, apresentando classificação da American Society Anesthesiologists (ASA) I ou II; a coleta de dados ocorreu de fevereiro a junho de 2013. Resultados: As complicações mais frequentes foram hipotermia, dor e hipoxemia. Houve associação estatisticamente significante entre o Índice de Aldrete Kroulik com bradipneia $(\mathrm{p}=0,038)$ e hipoxemia $(\mathrm{p}=0,025)$, na entrada do paciente na sala de recuperação pós-anestésica, e aos 60 minutos de permanência, com hipertensão arterial $(\mathrm{p}=0,023)$ e taquicardia $(\mathrm{p}=0,048)$. Conclusão: Diante dos resultados evidenciados, compete ao Enfermeiro a implementação de medidas eficazes na prevenção e no controle das complicações do paciente no período de recuperação anestésica.

PALAVRAS-CHAVE: Enfermagem perioperatória. Sala de recuperação. Complicações pós-operatórias.

ABSTRACT: Objective: To analyze patient complications during post-anesthesia recovery. Method: This non-experimental, comparative, prospective field study used a quantitative methodological approach. The sample consisted of 42 adults who had undergone elective surgery with general anesthesia, classified as I or II according to the American Society of Anesthesiologists (ASA). Data collection occurred from February to June 2013. Results: The most common complications were hypothermia, pain and hypoxemia. Statistically significant association was observed between the Aldrete Kroulik Index with bradipnea $(\mathrm{p}=0.038)$ and hypoxemia ( $\mathrm{p}=0.025)$, when patients entered the post-anesthesia recovery room; and with hypertension $(\mathrm{p}=0.023)$ and tachycardia $(\mathrm{p}=0.048)$, after 60 minutes in the room. Conclusion: Considering the findings, nurses should implement effective measures to prevent and manage patient complications during post-anesthesia recovery.

KEYWORDS: Perioperative nursing. Recovery room. Postoperative complications.

RESUMEN: Objetivo: analizar las complicaciones del paciente durante el período de recuperación anestésica. Método: estudio de abordaje metodológico cuantitativo, delineamiento no experimental, comparativo, de campo y prospectivo. La muestra se constituyó de 42 adultos, sometidos a cirugías electivas, con anestesia general, clasificada como I o II según los criterios de la American Society Anesthesiologists (ASA), y cuya recolección de datos ocurrió entre febrero y junio de 2013. Resultados: las complicaciones más frecuentes fueron hipotermia, dolor e hipoxemia. Hubo relación estadísticamente significativa del Índice de Aldrete Kroulik con bradipnea $(\mathrm{p}=0,038)$ e hipoxemia $(\mathrm{p}=0,025)$ cuando los pacientes entraban a la sala de recuperación post anestésica, y a los 60 minutos de permanencia, con hipertensión arterial $(\mathrm{p}=0,023)$ y taquicardia $(\mathrm{p}=0,048)$. Conclusión: ante los resultados evidenciados, compete al enfermero la implementación de medidas eficaces en la prevención y control de las complicaciones del paciente durante el período de recuperación anestésica.

PALABRAS CLAVE: Enfermería perioperatoria. Sala de recuperación. Complicaciones postoperatorias.

\footnotetext{
${ }^{1}$ Aluna do Curso de Graduação em Enfermagem. Universidade Federal de Minas Gerais. E-mail: fiamacn@yahoo.com.br ${ }^{2}$ Enfermeira. Doutora em Enfermagem. Professora do Departamento de Enfermagem Básica. Escola de Enfermagem. Universidade Federal de Minas Gerais. E-mail: selmesilqueira@gmail.com

${ }^{3}$ Enfermeira. Doutora em Enfermagem. Professora do Departamento de Enfermagem Básica. Escola de Enfermagem da Universidade Federal de Minas Gerais. Orientadora da pesquisa.

Avenida Professor Alfredo Balena, 190. Santa Efigênia. CEP 30130-100. Belo Horizonte, MG, Brasil.

Telefone: (31) 3409-9886. E-mail: almattia@uol.com.br

*Esta pesquisa teve o apoio financeiro da Fundação de Amparo à Pesquisa do Estado de Minas Gerais (FAPEMIG - APQ 655-13).
} 


\section{Introdução}

A Sala de Recuperação Pós-Anestésica (SRPA) é o local onde o paciente submetido ao procedimento anestésicocirúrgico deve permanecer, sob observação e cuidados constantes da Equipe de Enfermagem, até que haja recuperação da consciência, estabilidade dos sinais vitais, prevenção das intercorrências do período pós-anestésico e/ ou pronto atendimento ${ }^{1}$.

O período de Recuperação Anestésica (RA) é compreendido entre a alta da sala de operação (SO) até a alta da RA ${ }^{1}$. Este período deveria ocorrer em área física planejada, denominada SRPA, com equipe multiprofissional composta por Anestesiologista, Enfermeiro e Técnico/ Auxiliar de Enfermagem, treinada e habilitada a prestar cuidados individualizados de alta complexidade ${ }^{2}$.

No período de RA, o paciente fica vulnerável às complicações dos sistemas respiratório, cardiovascular, termorregulador, tegumentar, sensorial, locomotor, urinário, digestório e imunológico, além do estado emocional ${ }^{3-6}$. Desta forma, o paciente pode apresentar alterações da pressão arterial sistêmica, da frequência cardíaca e dos movimentos respiratórios, bem como temperaturas anormais, processo mental alterado, dor, náusea e vômito.

A ocorrência de complicações no paciente em SRPA está diretamente associada às condições clínicas pré-operatórias, à extensão e ao tipo de cirurgia, às intercorrências cirúrgicas e anestésicas, e à eficácia das medidas terapêuticas aplicadas ${ }^{4}$.

Devido à elevada incidência de complicações, é de extrema importância a permanência do paciente na SRPA até que o mesmo recobre a consciência, esteja com os reflexos protetores e sinais vitais estáveis; é também importante que, enquanto necessitar de cuidados especiais, sejam oferecidos ao paciente equipamentos de monitorização e equipe treinada para detectar precocemente estas alterações ${ }^{4}$.

Considerando-se a elevada incidência de complicações ao paciente no período de RA, levanta-se a seguinte questão: quais são as complicações mais frequentes ao paciente na SRPA?

Diante do exposto, esta pesquisa tem como objetivo analisar as complicações do paciente em período de RA.

\section{Método}

Trata-se de uma pesquisa com abordagem metodológica quantitativa, delineamento não experimental, comparativa, de campo e prospectiva.

Foi realizada em um hospital público, federal, de grande porte, localizado na capital do Estado de Minas Gerais. O campo da pesquisa foi a SRPA, que conta com oito leitos e onde mantêm-se a temperatura ambiente entre 22 e $24{ }^{\circ} \mathrm{C}$, e a umidade relativa do ar entre 45 e $60 \%$, conforme as recomendações do Ministério da Saúde 7 .

O projeto de pesquisa foi aprovado pelo Comitê de Ética em Pesquisa da Universidade Federal de Minas Gerais (CEP UFMG), atendendo à Resolução 466/2012 do Conselho
Nacional de Saúde, parecer do CEP UFMG 274.655 e CAAE 14887213.4.0000.5149.

O Termo de Consentimento Livre e Esclarecido (TCLE) foi assinado por todos os participantes após receberem do pesquisador as informações sobre a pesquisa e seus objetivos. Os esclarecimentos e a assinatura do termo foram realizados no quarto do paciente, quando este ainda se encontrava em fase pré-operatória.

O tamanho amostral foi definido segundo o número de variáveis preditivas inicialmente proposto, utilizando-se de dez a 11 sujeitos em relação a cada uma das variáveis8.

As variáveis preditivas foram: idade (adultos de 18 a 60 anos); momento operatório (cirurgia eletiva); tipo de anestesia (geral), e classificação física da American Society Anesthesiologists (ASA) I ou II, totalizando 42 sujeitos.

Desta forma, foram critérios de inclusão pacientes adultos, que assinaram o TCLE, com idade entre $18 \mathrm{e}$ 60 anos, submetidos à cirurgia eletiva, com anestesia geral e classificação de ASA I ou II.

Os critérios de exclusão foram pacientes com idade inferior a 18 anos ou superior a 60 anos, cirurgia de urgência e emergência, anestesia local ou regional, e classificação de ASA de III a VI.

A coleta de dados foi realizada de fevereiro a julho de 2013; para tal, foi elaborado um instrumento semiestruturado que contempla os seguintes dados: caracterização do paciente, caracterização do procedimento anestésico-cirúrgico e complicações apresentadas pelo paciente no período de RA.

Os dados coletados para caracterização do paciente foram sexo, idade, classificação de ASA e comorbidades préexistentes. O tipo e a duração caracterizaram o procedimento anestésico cirúrgico.

Para identificação e análise das complicações apresentadas pelos pacientes, foram coletados dados relativos ao Índice de Aldrete e Kroulik (IAK), sendo: atividade muscular, respiração, circulação, consciência e Saturação periférica de oxigênio $\left(\mathrm{SpO}_{2}\right)$.

A pontuação do IAK varia de 0 a 2 pontos para cada parâmetro, na qual o zero (0) indica condições de maior gravidade, a pontuação um (1) corresponde a um nível intermediário e a pontuação dois (2) representa as funções restabelecidas ${ }^{9}$.

De acordo com o IAK, a atividade muscular é avaliada com nota (2), quando o paciente movimenta os quatro membros; nota (1), quando movimenta dois membros, e nota (0), quando é incapaz de mover os membros voluntariamente ou sob comando. Este parâmetro foi utilizado para análise de alterações na movimentação.

A respiração é avaliada com nota (2), quando o paciente é capaz de respirar profundamente e tossir livremente; nota (1), na dispneia ou na limitação da respiração, e nota (0), na apneia ${ }^{6}$. Este parâmetro foi utilizado para análise das alterações na respiração, como dispneia, hipopneia e hiperpneia.

O parâmetro circulação é avaliado pela pressão arterial sistólica, comparada aos níveis pressóricos de pré-operatório, 
em que, na nota (2), a pressão arterial apresenta-se até $20 \%$ de diferença do nível pré-anestésico; a nota (1) indica pressão arterial com $20 \%$ a $49 \%$ de diferença do nível pré-anestésico, e nota $(0)$, quando pressão arterial apresentar diferença acima de $50 \%$ do nível pré-anestésico. Este parâmetro foi utilizado para análise de complicações pressóricas, como hipotensão e hipertensão arterial.

A consciência é avaliada com nota (2) se o paciente está lúcido, orientado no tempo e no espaço; nota (1), desperta se for solicitado, e nota (0), se não responde. Este parâmetro foi utilizado para análise das alterações do nível de consciência.

$\mathrm{Na} \mathrm{SpO}$, a nota (2) é atribuída ao paciente capaz de manter $\mathrm{SpO}_{2}>92 \%$, respirando ar ambiente; nota (1), se necessita de oxigênio suplementar para manter $\mathrm{SpO}_{2}>90 \%$, e nota (0), se $\mathrm{SpO}_{2}<90 \%$ mesmo com oxigênio suplementar ${ }^{6}$. Este parâmetro determinou a complicação da oxigenação, como a hipoxemia.

A aplicação do IAK na SRPA é indicada na chegada do paciente, considerado zero (0) minuto, de 15 em 15 minutos na primeira hora, de 30 em 30 minutos na segunda hora, e de hora em hora a partir da terceira hora de permanência na SRPA, ou seja, 0 (zero), 15, 30, 45, 60, 90, 120 e 180 minutos $^{6}$.

Os dados dos sinais vitais foram pressão arterial (PA), frequência cardíaca $(\mathrm{FC})$, frequência respiratória (FR) e temperatura corpórea axilar (T).

$\mathrm{Na}$ análise dos sinais vitais, para a PA, foi considerada hipotensão ou hipertensão arterial, a PA 20\% menor ou maior, respectivamente, do que a PA do nível pré-anestésico ${ }^{6}$.

Quanto à FC, considerou-se bradicardia, a FC menor do que 60 batimentos por minuto, e taquicardia, a FC maior do que 100 batimentos por minuto. Para análise da temperatura, foi considerada hipotermia a temperatura corporal menor do que $36^{\circ} \mathrm{C}$, e hipertermia, a temperatura acima de $37,8^{\circ} \mathrm{C}^{10}$.

A frequência respiratória foi considerada normal para adultos entre 12 e 22 incursões respiratórias por minuto (irpm) $)^{11}$.

A dor e a náusea foram analisadas pelo relato verbal do paciente; o vômito, pela presença deste.

Os dados foram coletados na admissão do paciente na SRPA, considerado como zero (0) minuto e, a partir deste, a cada 15 minutos, até completar 60 minutos.

A análise dos dados foi realizada pelo programa Statistical Package for Social Sciences (SPSS) 14.0. As variáveis categóricas foram apresentadas pelas frequências absolutas e relativas; as variáveis contínuas foram apresentadas com valores mínimo, máximo, média e desvio padrão. Foram aplicados os testes Kolmogorov-Smirnov e teste t de Student, utilizando-se o nível de significância de $5 \%(\mathrm{p} \leq 0,05)$.

\section{Resultados}

Os resultados estão apresentados com dados relativos à caracterização do paciente, ao procedimento anestésicocirúrgico e à caracterização das complicações apresentadas pelo paciente.

\section{Caracterização do paciente}

Nos dados sociodemográficos e clínicos, foram analisados o sexo, a idade, as comorbidades e a classificação de ASA.

Em relação ao sexo, houve prevalência do feminino, sendo este $28(66,7 \%)$ e $14(33,3 \%)$ do masculino; a média de idade foi de 40,3 anos, com desvio padrão $(12,5)$, acusando o mínimo de 18 anos e o máximo de 59 anos.

As comorbidades de maior frequência foram a Hipertensão Arterial Sistêmica e o Diabetes Mellitus, com 10 (23,8\%) e $9(21,4 \%)$ respectivamente. Na avaliação da condição física de ASA, houve prevalência da ASA II com 27 (64,3\%), e ASA I com 15 (35,7\%).

\section{Caracterização do procedimento anestésico- cirúrgico}

O procedimento anestésico-cirúrgico foi caracterizado pela duração da cirurgia e da anestesia, e pela especialidade cirúrgica.

A média de duração da cirurgia foi de 165,6 minutos, com desvio padrão $(88,5)$, com o máximo de 480 minutos e o mínimo de 30 minutos. A duração da anestesia foi de 211,9 minutos, com desvio padrão $(83,6)$, com máximo de 530 minutos e mínimo de 75 minutos.

Foi critério de inclusão, neste estudo, o tipo de anestesia; assim, todos os sujeitos receberam anestesia geral, enquanto que a especialidade cirúrgica de maior frequência foi Ginecologia- 12 (28,5\%), seguida de Urologia- 9 (21,4\%), Gastroenterologia- 7 (16,6\%), Otorrinolaringologia- 7 $(16,6 \%)$, Ortopedia e Traumatologia- 4 (9,5\%), e Cirurgia Plástica- 3 (7,1\%).

\section{Caracterização das complicações apresentadas pelo paciente}

As complicações apresentadas pelo paciente foram analisadas por meio dos valores dos sinais vitais, do IAK e de manifestações e relatos de dor, náuseas e vômitos. Estes parâmetros foram analisados na chegada do paciente na SRPA, considerada zero (0) minuto, e a partir deste, a cada 15 minutos, até completar 60 minutos.

A Tabela 1 é referente aos parâmetros dos sinais vitais (PA, FC, FR e T) em 0, 15, 30, 45 e 60 minutos. Observa-se que, em relação ao tempo de permanência na SRPA, as médias de PA e FC diminuíram, as médias de FR mantiveram-se praticamente constantes e as médias de $\mathrm{T}$ apresentaram um aumento.

No que se refere aos parâmetros do IAK - atividade muscular, respiração, circulação, consciência e $\mathrm{SpO}_{2}$-, estes também foram analisados a cada 15 minutos na SRPA. As pontuações do IAK variaram entre dois (2) e um (1), sendo que nenhum paciente obteve nota zero (0).

Quanto à atividade muscular, todos os pacientes -42 $(100,0 \%)$ - tiveram pontuação dois (2), desde a chegada na SRPA até completar 60 minutos de permanência. 
Tabela 1. Distribuição da frequência dos valores dos sinais vitais. Belo Horizonte, 2013.

\begin{tabular}{|c|c|c|c|}
\hline Sinais vitais & Média (dp) & Mínimo & Máximo \\
\hline \multicolumn{4}{|l|}{$\mathrm{PA}(\mathrm{mmHg})$} \\
\hline 0 & $120,0(17,2)$ & 92 & 164 \\
\hline 15 & $116,5(14,3)$ & 89 & 145 \\
\hline 30 & $116,9(15,1)$ & 87 & 171 \\
\hline 45 & $117,1(15,1)$ & 81 & 157 \\
\hline 60 & $115,6(16,1)$ & 83 & 153 \\
\hline \multicolumn{4}{|l|}{ FC (bat/min) } \\
\hline 0 & $85,7(15,4)$ & 54 & 118 \\
\hline 15 & $83,9(17,9)$ & 53 & 129 \\
\hline 30 & $80,1(14,5)$ & 55 & 114 \\
\hline 45 & $79,7(14,2)$ & 54 & 112 \\
\hline 60 & $79,8(14,9)$ & 54 & 116 \\
\hline \multicolumn{4}{|l|}{ FR (irpm) } \\
\hline 0 & $16,2(1,6)$ & 10 & 20 \\
\hline 15 & $16,4(1,7)$ & 10 & 20 \\
\hline 30 & $16,3(1,5)$ & 10 & 20 \\
\hline 45 & $16,4(1,6)$ & 10 & 20 \\
\hline 60 & $16,3(1,6)$ & 10 & 20 \\
\hline \multicolumn{4}{|l|}{$\mathrm{T}\left({ }^{\circ} \mathrm{C}\right)$} \\
\hline 0 & $34,8(0,9)$ & 32,7 & 36,5 \\
\hline 15 & $35,0(0,8)$ & 33,1 & 36,5 \\
\hline 30 & $35,2(0,8)$ & 33,4 & 36,7 \\
\hline 45 & $35,3(0,7)$ & 33,4 & 36,7 \\
\hline 60 & $35,5(0,8)$ & 33,1 & 36,7 \\
\hline
\end{tabular}

A respiração manteve-se com a mesma avaliação durante os 60 minutos, sendo 7 pacientes $(16,7 \%)$ com pontuação um (1) e 35 pacientes $(83,3 \%)$ com pontuação dois (2).

No parâmetro da circulação, 13 pacientes $(31,0 \%)$ chegaram com pontuação 1 (um) e, destes, aos 60 minutos, 11 pacientes $(26,2 \%)$ permaneceram com esta pontuação.

Quanto ao nível de consciência, 17 pacientes (40,5\%) chegaram sonolentos, com pontuação de um (1), e aos 60 minutos, 5 pacientes $(11,9 \%)$ mantiveram-se na mesma condição.

$\mathrm{Na}$ análise da $\mathrm{SpO}_{2}, 19$ pacientes $(45,2 \%)$ chegaram na SRPA com pontuação um (1), e aos 60 minutos, 31 pacientes $(73,8 \%)$ estavam com a pontuação dois (2).

A Tabela 2 demonstra os valores do IAK em relação ao tempo de permanência do paciente na SRPA. Observa-se a melhora da média da pontuação a cada 15 minutos de permanência.

As complicações apresentadas pelos pacientes em período de RA foram hipotensão e hipertensão arterial, bradicardia e taquicardia, bradipneia, hipotermia, alteração na respiração, hipoxemia, alteração do nível de consciência, náusea, vômito e dor (Tabela 3).
Tabela 2. Distribuição da frequência dos valores do IAK. Belo Horizonte, 2013.

\begin{tabular}{cccc}
\hline Tempo & Média (dp) IAK & Mínimo & Máximo \\
\hline 0 & $8,4(1,21)$ & 6 & 10 \\
15 & $8,6(1,02)$ & 6 & 10 \\
30 & $8,8(1,04)$ & 6 & 10 \\
45 & $9,0(1,09)$ & 6 & 10 \\
60 & $9,0(0,99)$ & 7 & 10 \\
\hline
\end{tabular}

Observa-se na Tabela 3 que as complicações mais frequentes foram hipotermia, dor e hipoxemia. Ao longo de 60 minutos, a média para hipotermia foi de 33,6 pacientes e, para dor e hipoxemia, foi de 19,0 e 16,0 pacientes, respectivamente.

Nenhum paciente apresentou complicação na movimentação, taquipneia e hipertermia.

Os pacientes apresentaram um decréscimo, ao longo dos 60 minutos de permanência na SRPA, determinadas alterações, como hipotensão arterial, taquicardia, bradipneia, na respiração, no nível de consciência e náusea.

Observa-se que houve acréscimo na frequência da bradicardia e na dor, ao longo do tempo, e que a frequência da hipotensão arterial foi relativamente constante.

Quanto à hipotermia, 30 pacientes $(71,4 \%)$ mantiveram-se hipotérmicos ao longo de 60 minutos de permanência em SRPA.

A Tabela 4 apresenta a associação entre o IAK e as alterações apresentadas pelos pacientes, na entrada e após 60 minutos de permanência na SRPA.

$\mathrm{Na}$ entrada do paciente, em zero (0) minuto, houve significância estatística entre o IAK e a bradipneia $(p=0,038)$ e a hipoxemia $(p=0,025)$. Aos 60 minutos, a bradipneia e a hipoxemia mantiveram a significância estatística com o IAK, e ainda, aos 60 minutos, a hipertensão arterial e a taquicardia ( $\mathrm{p}=0,023$ e 0,048 , respectivamente) apresentaram significância estatística.

\section{Discussão}

Os resultados desta pesquisa evidenciaram que as complicações apresentadas pelos pacientes, em período de RA, foram hipotensão e hipertensão arterial, bradicardia e taquicardia, bradipneia, hipotermia, alteração na respiração, hipoxemia, alteração do nível de consciência, náusea, vômito e dor. Destas, as mais frequentes foram a hipotermia, a dor e a hipoxemia.

A identificação destas complicações foi realizada pela análise dos sinais vitais e do IAK, pelo relato de dor e pela presença de náusea e vômito

A hipotermia foi a complicação mais frequente, sendo que, em média, 33,6 pacientes apresentaram hipotermia na primeira hora de permanência na SRPA. A hipotermia, definida como temperatura central corporal menor do que 
Tabela 3. Distribuição da frequência das complicações apresentadas pelos pacientes. Belo Horizonte, 2013.

\begin{tabular}{|c|c|c|c|c|c|c|}
\hline \multirow{2}{*}{ Variáveis } & 0 & 15 & 30 & 45 & 60 & Média \\
\hline & $\mathrm{N}(\%)$ & $\mathrm{N}(\%)$ & $\mathrm{N}(\%)$ & $\mathrm{N}(\%)$ & $\mathrm{N}(\%)$ & $(\mathrm{dp})$ \\
\hline Hipotensão arterial & $7(16,7)$ & $8(19,0)$ & $7(16,7)$ & $8(19,0)$ & $8(19,0)$ & $7,6(1,02)$ \\
\hline Hipertensão arterial & $6(14,3)$ & $3(7,1)$ & $3(7,1)$ & $2(4,8)$ & $3(7,1)$ & $3,4(1,03)$ \\
\hline Bradicardia & $2(4,8)$ & $4(9,5)$ & $5(11,9)$ & $4(9,5)$ & $5(11,9)$ & $4,0(1,04)$ \\
\hline Taquicardia & $9(21,4)$ & $8(19,0)$ & $2(4,8)$ & $2(4,8)$ & $3(7,1)$ & $4,8(1,23)$ \\
\hline Bradipneia & $9(21,4)$ & $8(19,0)$ & $3(7,1)$ & $2(4,8)$ & $2(4,8)$ & $4,8(1,22)$ \\
\hline Hipotermia & $35(83,3)$ & $35(83,3)$ & $35(83,3)$ & $33(78,6)$ & $30(71,4)$ & $33,6(1,05)$ \\
\hline Alteração na respiração & $12(28,6)$ & $7(16,7)$ & $5(11,9)$ & $5(11,9)$ & $5(11,9)$ & $6,8(1,25)$ \\
\hline Hipoxemia & $19(45,2)$ & $20(47,6)$ & $17(40,4)$ & $13(30,9)$ & $11(26,1)$ & $16,0(1,03)$ \\
\hline Alteração da consciência & $17(40,5)$ & $9(21,4)$ & $7(16,7)$ & $6(14,3)$ & $4(9,5)$ & $8,6(1,34)$ \\
\hline Dor & $16(38,1)$ & $18(42,9)$ & $18(42,9)$ & $21(50,0)$ & $22(52,4)$ & $19,0(1,03)$ \\
\hline
\end{tabular}

Tabela 4. Associação do IAK e as complicações apresentadas pelos pacientes na entrada e aos 60 minutos de permanência na SRPA. Belo Horizonte, 2013.

\begin{tabular}{|c|c|c|}
\hline Variáveis & 0 & 60 \\
\hline \multicolumn{3}{|l|}{ IAK } \\
\hline Hipotensão arterial & 0,584 & 0,485 \\
\hline \multicolumn{3}{|l|}{ IAK } \\
\hline Hipertensão arterial & 0,760 & 0,023 \\
\hline \multicolumn{3}{|l|}{ IAK } \\
\hline Bradicardia & 0,825 & 0,671 \\
\hline \multicolumn{3}{|l|}{ IAK } \\
\hline Taquicardia & 0,463 & 0,048 \\
\hline \multicolumn{3}{|l|}{ IAK } \\
\hline Bradipneia & 0,038 & 0,024 \\
\hline \multicolumn{3}{|l|}{ IAK } \\
\hline Hipotermia & 0,600 & 0,721 \\
\hline \multicolumn{3}{|l|}{ IAK } \\
\hline Alteração na respiração & 0,350 & 0,563 \\
\hline \multicolumn{3}{|l|}{ IAK } \\
\hline Hipoxemia & 0,025 & 0,015 \\
\hline \multicolumn{3}{|l|}{ IAK } \\
\hline Alteração do nível da consciência & 0,774 & 0,674 \\
\hline \multicolumn{3}{|l|}{ IAK } \\
\hline Náusea/Vômito & 0,934 & 0,874 \\
\hline \multicolumn{3}{|l|}{ IAK } \\
\hline Dor & 0,301 & 0,423 \\
\hline
\end{tabular}

$\mathrm{p} \leq 0,05$.

$36{ }^{\circ} \mathrm{C}$, é um evento comum ao paciente submetido a este processo $^{12-16}$.

Estudos apontam que pacientes submetidos ao procedimento anestésico-cirúrgico estão expostos a vários fatores, que podem alterar a termorregulação; dentre os fatores que contribuem para esta complicação, podemos destacar a temperatura da SO; a infusão de soluções frias em cavidades ou por via endovenosa; a idade dos pacientes; o relaxamento muscular; a exposição de cavidades; o tempo de cirurgia; o tipo de cirurgia, e a ventilação com gases não aquecidos ${ }^{9,12-16}$.

O controle da hipotermia é de extrema importância para a Enfermagem por este quadro acarretar não apenas um desconforto térmico ao paciente, mas também causar outras complicações, tais como: aumento da morbidade cardíaca; arritmias; elevação das catecolaminas; alterações hormonais; coagulopatias; aumento da infecção de sítio cirúrgico, bem como prolongamento da recuperação do paciente ${ }^{12-15}$.

As medidas para a prevenção de hipotermia, em especial as indiretas, devem ser planejadas e implementadas pelo Enfermeiro, iniciando-se no período pré-operatório e prolongando-se até a alta do paciente da SRPA ${ }^{16}$.

Nesta pesquisa, a dor foi a segunda complicação de maior frequência, sendo que, em média, 19,0 pacientes $(45,2 \%)$ apresentaram dor na primeira hora de permanência na SRPA. A dor é umas das complicações mais comuns na SRPA e deve ser tratada prontamente, uma vez que pode ser responsável por desconforto, agitação, alterações hemodinâmicas e prolongamento da hospitalização $0^{17,18}$.

$\mathrm{O}$ tratamento da dor baseia-se em razões subjetivas, de forma a atenuar as respostas físicas e psicológicas do trauma cirúrgico ${ }^{14}$. Alguns autores afirmam que a prevenção da dor pós-operatória é fundamental para melhor resultado anestésico-cirúrgico, bem como para diminuir o sofrimento e o trauma associados ao período de permanência hospitalar ${ }^{13-15}$.

A hipoxemia foi a complicação mais frequente depois da hipotermia e da dor, sendo apresentada, em média, por 16,0 pacientes. Foi analisada por meio da $\mathrm{SpO}_{2}$ atribuindo-se nota (1) conforme o IAK, se o paciente necessita de oxigênio suplementar para manter $\mathrm{SpO}_{2}>90 \%$.

A hipoxemia, na maioria das vezes, está relacionada à anestesia. O paciente pode apresentar depressão respiratória 
pela ação residual de opióides e bloqueadores neuromusculares, pela perda de reflexos vasoconstritores, pelo aumento de consumo de oxigênio e pelos tremores musculares, fato que pode ocasionar, dentre outros, sonolência, e aumentar o tempo para recuperação e alta da SRPA $^{14}$.

A American Society of PeriAnesthesia Nurses (ASPAN) recomenda a admissão do paciente na SRPA, sistematizada em três etapas. A primeira etapa é denominada avaliação do $\mathrm{ABC}$, sendo Airway (vias aéreas), Breathing (respiração) e Circulation (circulação). Na avaliação das vias aéreas, as intervenções recomendadas são a observação da perviedade, a administração de oxigênio umidificado e a colocação da oximetria de pulso, com a finalidade de prevenção de hipoxemia ${ }^{19}$.

As alterações da pressão arterial foram analisadas pelo parâmetro circulação do IAK, em que se atribuiu nota (1) para pressão arterial com $20 \%$ a $49 \%$ de diferença do nível pré-anestésico ${ }^{9}$. Em média 7,6 e 3,4 pacientes apresentaram hipotensão e hipertensão arterial, respectivamente.

As alterações para mais ou para menos de $20 \%$ dos valores pressóricos basais constituem as complicações de hipertensão e hipotensão arterial, respectivamente. Dentre os fatores que contribuem para a hipertensão arterial na SRPA, podemos destacar aqueles ligados a uma hipertensão arterial de base, bem como fatores associados a dor, medo, realização de inspirações profundas, curativo e associação de fármacos ${ }^{3-6,14}$.

As variações pressóricas podem ocorrer em sequências distintas do ato cirúrgico, podendo elevar-se durante a indução anestésica, diminuir com o aprofundamento da anestesia e aumentar novamente no período da recuperação ${ }^{13,14}$.

Os fatores que contribuem para a hipotensão arterial podem estar associados à hidratação inadequada durante o período anestésico-cirúrgico e aos efeitos da anestesia, bem como às disfunções cardíacas, como infarto do miocárdio, tamponamento, embolia ou medicação, incluindo, nestes, os agentes anestésicos ${ }^{5,6,13}$.

Ahipotensão arterialé experimentada por aproximadamente 3\% dos pacientes no pós-operatório. Dentre os sinais clínicos desta complicação, destacamos pulso rápido e filiforme, desorientação, sonolência, oligúria, pele fria e pálida, sendo, desta maneira, imprescindível a estes pacientes uma avaliação do Enfermeiro, visto que, para esta complicação, os sinais clínicos são o indicador mais confiávele ${ }^{2-6}$.

Nesta pesquisa, a média de pacientes que apresentou bradicardia e taquicardia foi de 4,0 e 4,8 pacientes, respectivamente. A taquicardia e a bradicardia, caracterizadas por valores de batimentos cardíacos por minuto superiores a 100 e inferiores a 60, respectivamente, têm relação direta com presença de dor, hipoxemia, hipotermia, aumento da temperatura e ansiedade $\mathrm{e}^{3,6,14}$.

Em média, 4,8 pacientes apresentaram bradipneia. A bradipneia é caracterizada por um número de incursões respiratórias inferior a 12 , durante um período de um minuto, sendo considerada normal de 12 a 22 irpm $^{10,11}$.
A presença da bradipneia no período de recuperação pós-anestésica está relacionada ao efeito residual da anestesia, à presença de dor e ao medo associado à realização de inspiração devido à dor, e à hipotermia, podendo gerar complicações, como a hipoventilação e a hipoxemia ${ }^{14}$.

A alteração na respiração foi analisada pelo IAK, atribuindo-se nota (1) na dispneia ou na limitação da respiração. Em média, 6,8 pacientes apresentaram esta complicação. Tal complicação pode estar intimamente associada às outras complicações já citadas, tais como bradipneia, taquicardia, bradicardia, dor e ansiedade, e pode levar, dentre outras, a consequências como a hipoxemia e a alteração do nível de consciência.

A alteração do nível de consciência foi observada, em média, em 8,6 pacientes. Está associada à sintomatologia em que o paciente pode apresentar-se desorientado, sonolento, confuso ou delirante. Geralmente, esta complicação está relacionada, principalmente, ao efeito residual da anestesia, à hipoxemia, à dor ou à ansiedade ${ }^{2-4}$.

Mesmo com o avanço da tecnologia, com aprimoramento de técnicas cirúrgicas e utilização de novas gerações de antieméticos e anestésicos, a náusea e o vômito ainda aparecem como complicação nos pacientes na SRPA ${ }^{12,18}$. Nesta pesquisa, a média desta complicação foi 0,2 paciente, ou seja, apenas um paciente apresentou náusea na entrada da SRPA.

O controle desta complicação deve se iniciar no período pré-operatório e deve continuar durante todo o período intraoperatório, visto que os fatores causais desta complicação estão relacionados diretamente ao processo anestésico-cirúrgico ${ }^{2-4,14}$.

Além da provisão e do gerenciamento de recursos, cabe ao profissional Enfermeiro identificar as complicações dos pacientes na SRPA, a fim de implementar ações que evitem ou minimizem as complicações do paciente durante o processo cirúrgico. Tendo em vista que este profissional e sua equipe são responsáveis do ponto de vista profissional, ético e legal pelo paciente nos diferentes períodos da cirurgia, é imprescindível que as ações deste sejam planejadas para todo o período pré-operatório, implementadas no transoperatório e avaliadas por todo o período perioperatório ${ }^{13,20}$.

\section{Conclusão}

Esta pesquisa permitiu concluir que as complicações apresentadas pelos pacientes em período de RA foram hipotensão e hipertensão arterial, bradicardia e taquicardia, bradipneia, hipotermia, alteração na respiração, hipoxemia, alteração do nível de consciência, náusea, vômito e dor. As complicações mais frequentes foram hipotermia, dor e hipoxemia.

Os dados analisados demonstraram que houve significância estatística ente o IAK e bradipneia e hipoxemia, na entrada do paciente, e aos 60 minutos de permanência, houve 
significância entre o IAK com bradipneia, hipoxemia, hipertensão arterial e taquicardia.

Das complicações analisadas, algumas apresentaram um decréscimo ao longo do tempo de permanência do paciente na SRPA, como hipotensão arterial, taquicardia, bradipneia, alteração na respiração, alteração do nível de consciência e náusea; entretanto, outras, como bradicardia e dor, apresentaram um acréscimo. A hipotermia, mesmo com decréscimo, após os 60 minutos, $71,4 \%$ dos pacientes ainda apresentavam-se hipotérmicos.

Sugere-se que, na SRPA, sejam utilizadas medidas de monitoramento das complicações, como aplicação do IAK, monitorização cardíaca e da saturação periférica de oxigênio, e avaliação dos sinais vitais, dor, náuseas e vômitos.

Recomenda-se especial atenção para o tempo de permanência do paciente na SRPA, que deve ser, no mínimo, de 60 minutos, para que se possa avaliar a evolução das potenciais complicações.

Diante dos resultados evidenciados, compete ao Enfermeiro a implementação de medidas eficazes no controle das complicações do paciente, no período de recuperação anestésica.

\section{Referências}

1. Castellanos BE, Jouclas VM. Assistência de enfermagem perioperatória-um modelo conceitual. Rev Esc Enferm USP. 1990;24(3):359-69. PMid:2082439.

2. Cunha ALSM, Peniche ACG. Validação de um instrumento de registro para sala de recuperação pós-anestésica. Acta Paul Enferm. 2007;20(2):151-60. http://dx.doi.org/10.1590/ S0103-21002007000200007.

3. Galdeano LE, Rossi LA, Peniche ACG. Assistência de enfermagem na recuperação pós-anestésica. In: Carvalho R, Bianchi ERF. Enfermagem em centro cirúrgico e recuperação. Barueri: Manole; 2007. p. 267-98.

4. de Moraes LO, Peniche AC. Assistência de enfermagem no período de recuperação anestésica: revisão de literatura. Rev Esc Enferm USP. 2003;37(4):34-42. http://dx.doi.org/10.1590/S008062342003000400004. PMid:14727442

5. Popov DC, Peniche AC. [Nurse interventions and the complications in the post-anesthesia recovery room]. Rev Esc Enferm USP. 2009;43(4):953-61. http://dx.doi.org/10.1590/S008062342009000400030. PMid:20085169

6. Associação Brasileira de Enfermeiros de Centro Cirúrgico, Recuperação Anestésica e Centro de Material e Esterilização SOBECC. Práticas Recomendadas SOBECC. 6. ed. São Paulo: SOBECC; 2013.
7. Brasil. Ministério da Saúde. Portaria ${ }^{\circ} 1.884$, de 11 de novembro de 1994. Normas para Projetos Físicos de Estabelecimentos Assistenciais de Saúde. Brasília: Ministério da Saúde; 1994.

8. Chatterjee S, Hadi AS. Regression analysis by example. New Jersey: John Wiley \& Sons; 2006.. http://dx.doi.org/10.1002/0470055464.

9. de Castro FS, Peniche AC, Mendoza IY, Couto AT. Temperatura corporal, índice Aldrete e Kroulik e alta do paciente da Unidade de Recuperação Pós-Anestésica. Rev Esc Enferm USP. 2012;46(4):872-6. http://dx.doi.org/10.1590/S008062342012000400013. PMid:23018396

10. Potter PA, Perry AG. Fundamentos de enfermagem. 6. ed. Rio de Janeiro: Elsevier; 2005.

11. Barros ALBL, organizadora. Anamnese e exame físico: avaliação diagnóstica de enfermagem no adulto. 2. ed. São Paulo: Artmed; 2010.

12. Poveda VB, Galvão CM. Hipotermia no período intra-operatório: é possível evitá-la? Rev Esc Enferm USP. 2011;45(2):411-7. http:// dx.doi.org/10.1590/S0080-62342011000200016. PMid:21655792

13. de Mattia AL, Faria Maia L, Santos Silva S, de Oliveira TC. Diagnósticos de enfermagem nas complicações em sala de recuperação anestésica. Enfermería Global [Internet]. 2010 [acesso em 2013 Ago 08];9(18). Disponível em: http://revistas.um.es/ eglobal/article/view/93601/90161

14. Popov DC, Peniche AC. As intervenções do enfermeiro e as complicações em sala de recuperação pós-anestésica. Rev Esc Enferm USP. 2009;43(4):953-61. http://dx.doi.org/10.1590/S008062342009000400030. PMid:20085169

15. Capello RG, Alves ALS, Cézar Júnior A, Carvalho R. Intervenções de enfermagem na recuperação anestésica: controle da dor, náuseas, hipotermia e outras complicações do pós-operatório. Rev Dor. 2009;10(2):113-9.

16. De Mattia AL, Barbosa MH, Freitas Filho JPA, Rocha AM, Pereira NHC. Infusão venosa aquecida no controle da hipotermia no período intraoperatório. Rev Latino-Am Enfermagem. 2013;21(3):801-10.

17. De Mattia AL, Silva AG, Arantes SG. Atuação do enfermeiro no controle da dor em recuperação anestésica. Rev SOBECC. 2008;13(3):27-32.

18. Mendonza IYQ, Peniche ACG. Complicações do paciente cirúrgico idoso no período de recuperação pós-anestésica: revisão de literatura. Rev SOBECC. 2008;13(1):25-31.

19. American Society of PeriAnesthesia Nurses - ASPAN. Perianesthesia nursing standarts, practice recommendations and interpretative statements. Cherry Hill: ASPAN; 2012-14.

20. Mendonza IYQ, Freitas GF, Oguisso T, Peniche ACGP. Retrospectiva histórica das salas de recuperação pós-anestésica em enfermagem. Temperamentvm [Internet]. 2010 [acesso em 2010 Set 28];11. Disponível em: http://www.index f.com/ temperamentum/tn11/t7186.php 